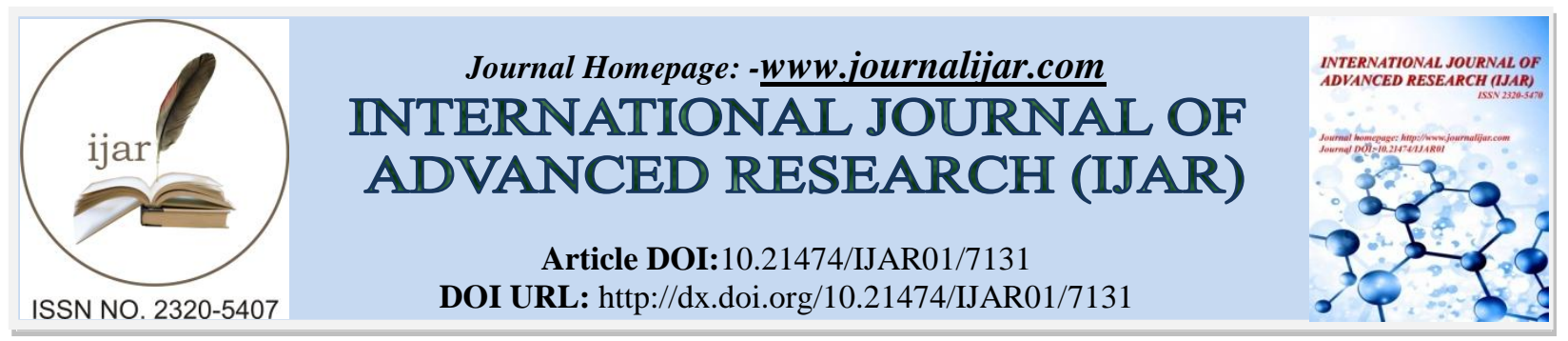

RESEARCH ARTICLE

\title{
REFLEXION SUR LA POLYGAMIE MAROCAINE FACE AU DROIT INTERNATIONAL PRIVE FRANÇAIS.
}

Dr. Leilla ben sedrine.

Professeur chercheur à la Faculté des Sciences Juridiques Economiques et Sociales, Souissi- Université Mohamed V, Rabat. Maroc.

\section{Manuscript Info}

Manuscript History

Received: 18 March 2018

Final Accepted: 20 April 2018

Published: May 2018

Keywords:-

Polygamy - family law privateinternational law -conflicts of laws - conflicts of jurisdictions foreigner - national law - host country public policy.

\begin{abstract}
The polygamy in Morocco is a subject which gives to reflection especially when it is confronted with a layman institution like France where this practice is totally prohibited. Thus the evaluation of the incompatibility of the Moroccan institution regarding polygamy with the requirements of the French legal system is taxable. So, takes place in the center of the debate the questions of conflicts of laws concerning franco-moroccan polygamous marriage leading in its path of doubts as for the capacity of the French legal system to govern foreign institution that some believe to be incompatible withpublic policy.
\end{abstract}

Copy Right, IJAR, 2018,. All rights reserved.

\section{Introduction:-}

La question de la polygamie est une question extrêmement sensible surtout lorsqu'elle est légale dans un pays et interdite dans l'autre. Dans l'expérience juridique française, les situations de polygamie ne sont pas nouvelles, elles sont nombreuses et comprennent un grand nombre de pays africains vu l'importance du flux migratoire.

Le Maroc en est bien l'illustration. Un pays régit par un statut personnel qui reconnait bien la polygamie mais il est confronté à un système français laïc qui refuse la polygamie et la considère comme une infraction pénale.

C'est pour cela que notre réflexion sera en grande partie consacrée aux différents problèmes juridiques que peut rencontrer le mariage polygamique marocain face au droit international français, à la limitation des effets de la polygamie par le droit des étrangers et les conséquences qui puissent en découlent ainsi qu'aux solutions envisagées par les deux systèmes, français et marocain en matière de conflits de lois,vu la spécificité des deux législations en matière de statut personnel.

\section{I- La Polygamie Et Son Cadre Legal :-}

\section{A-Définition de la polygamie en général}

La polygamie est un terme qui est formé, à partir de deux mots grecs, polus qui signifie «plusieurs» et gamosqui veut dire «mariage ». C'est également un terme générique qui recouvre plusieurs réalités. Elle peut premièrement 
être de jureoude facto. ${ }^{1}$ De jure, inclut la polyandrie, le fait d'une femme mariée à plusieurs hommes, ainsi que la polygynie, le fait d'un homme marié à plusieurs femmes qui est la forme la plus répandue et où le caractère patriarcal de l'institution et ses effets envers les femmes constituent les obstacles principaux à son acceptation en Occident.

Elle demeure pratiquée par plusieurs peuples selon des fondements religieux, notamment mormons ${ }^{2}$ et musulmans, ou coutumiers, en Afrique ou en Chine par exemple. ${ }^{3}$

Pour les musulmans, la polygamie est une situation qui permet à un homme de se marier au même temps, de plus qu'une épouse à condition que le nombre ne dépasse quatre conformément à ce qui a été prévu par le texte coranique. ${ }^{4}$ Une culture qui est rejeté moralement par une institution française qui ne voit en elle aucun respect au principe de l'égalité entre homme et femme mais aussi une violation à l'ordre public, dans la mesure où «l'Islam, avec sa polygamie, fût-elle théorique, est plus étranger à notre âme que n'importe quel autre système de droit ».5,

\section{1-Distinction entre polygamie et monogamie}

La monogamie est un mariage d'un homme et d'une femme sans possibilité pour l'un ou pour l'autre de célébrer un second mariage sans la dissolution du premier.L'article147 du code civil français stipule clairement qu' «on ne peut contracter un second mariage avant la dissolution du premier $\gg{ }^{6}$

\section{2-Distinction entre polygamie et bigamie}

Le terme «polygamie » est souvent confondu avec celui de «bigamie » : l'époux bigame est celui qui contracte un second mariage avant la dissolution du précédent alors que sa loi personnelle lui impose la monogamie. En revanche, l'époux polygame est celui dont les mariages simultanés sont autorisés par son statut personnel.

\section{B-Le cadre légal de la polygamie}

C'est la loi nationale de chaque pays qui permettra de définir si une personne est de statut personnel polygamique ou de statut personnel monogamique.

\section{1-La polygamie au Maroc}

S'inspirant des sources pures du droit musulman et du rite malikite, le mariage polygamique est intégré dans le corps même du Code régissant la famille au Maroc. Dans l'ancien Code de statut personnel,la polygamie était une institution qui permettait à l'époux par sa seule volonté de prendre une deuxième épouse sans avoir à rendre de comptes à sa première épouse.

Aujourd'hui et avec la promulgation du Code de la famille en 2004 (Moudawana), ${ }^{7}$ la polygamie est soumise à une série de restrictions sévèreset à un contrôle du juge. Elle est bien encadréede manière à offrir plus de protection à la femme. Ainsi, leCode de la famillen'autorise pas la polygamie dans ce genre de situations:

Lorsque le demandeur ne dispose pas de ressources suffisantes pour pourvoir aux besoins des deux foyers et leur assurer équitablement, l'entretien, le logement et les autres exigences de la vie. (Art 41 du Code de la famille). ${ }^{8} \mathrm{Il}$ doit être capable d'assurer l'égalité entre elles dans tous les aspects de la vie. ${ }^{9}$

1- De facto, elle inclut en surplus la notion de polyamorie, soit le fait d'arrangements relationnels variés comprenant des hommes et des femmes de toute orientation sexuelle : Maura I. Strassberg.; "The Challenge of PostModernPolygamy: ConsideringPolyamory » (2003) 31 Capital U.L. Rev. 439 à la p. 440.

2 -Secte située essentiellement aux États-Unis croyant au mariage polygamique (polygynie).

3 - Esther M. Kisaakye, «Women.; Culture and HumanRights: FemaleGenital Mutilation, Polygamy and Bride Price » dans Wolfgang Benedek - Esther M. Kisaakye et GerdOberleitner, dir. ;The HumanRights of Women: Iinternational Instruments and AfricanExperiences, Londres, World University Service, 2002, 268 à la p. 277.

${ }^{4}$ - Le verset 3 de Sourate les femmes précise « ... il est permis d'épouser deux, trois ou quatre, parmi les femmes qui vous plaisent... ».

5 -J.CARBONNIER. ; «Terre et ciel dans le droit français du mariage », in mélanges Ripert, Dalloz, 1950, p.341.

${ }^{6}$ - Code civil français - Livre $1^{\text {er }}$ des personnes, titre V du mariage. Créé par la loi n ${ }^{\circ} 1803-03-17$ promulguée le 27 mars 1803.

7 - Dahir n ${ }^{\circ}$ 1-04-22 du 12 hija 1424 (3 Février 2004) portant promulgation de la loi n ${ }^{\circ} 70-03$ portant Code de la famille. 
Ainsi et selon l'article 40 du Code de la famille: «La polygamie n'est pas autorisée s'il est craint que justice ne soit faite entre les époux $\gg .{ }^{10}$

Par ailleurs, si le mari est autorisé à prendre une autre épouse, le mariage avec celle-ci ne peut être conclu qu'après qu'elle ait été informée par le juge que le prétendant est déjà marié et qu'elle ait exprimé son consentement.

Dans la pratique, la tendance des hommes marocains à la polygamie reste curieusement très insignifiante par rapport aux autres pays islamiques ${ }^{11}$, cependant bien qu'elle se fait de plus en plus rare, elle garde toute sa place dans les pages du Code de la famille marocainet continue d'être pratiquée au sein du Maroc ainsi qu'à l'étranger en l'occurrence la France.

\section{2-La polygamie en France}

La polygamie en France est formellement interdite. Cette prohibition remonte au Code Napoléon de 1804 dans la mesure où un tel mariage heurtait la conception française de l'ordre public international et cela en portant atteinte à «l'égalité entre époux », principe ayant une grande valeur constitutionnelle en France.

La polygamie est interdite par l'article 147 du Code civil et est soumise à une sanction assez rigoureuse prévue par l'article 433-20 du Code pénal français qui stipule clairement : «Le fait, pour une personne engagée dans les liens du mariage, d'en contracter un autre avant la dissolution du précédent, est puni d'un an d'emprisonnement et de 45.000 euros d'amende.Est puni des mêmes peines l'officier public ayant célébré ce mariage en connaissant l'existence du précédent $\gg .{ }^{12}$

Ainsi, le fait de contracter un mariage avant la dissolution d'une précédente union valablement célébrée, est la définition même de la bigamie. Cette dernière constitue un délit pénal qui ne peut exister que dans le cadre d'un système monogame. Donc par hypothèse, ce n'est pas la polygamie qui constitue un délit mais la bigamie. ${ }^{13}$

Ceci dit, la France est confrontée à un flux migratoire important de marocains dont certains d'entre eux sont polygames. Doit-on comprendre que la loi française admet les unions polygamiques et leurs effets sur son territoire au nom du respect des cultures, ou les refuser au risque de porter atteinte aux droits des femmes et de nier l'effectivité d'une situation juridique étrangère?

Certes, la loi française admet l'union polygamique sur son territoire, une union qui doit respecter les particularités du Code civil français.

En effet, selon l'article 3 alinéa 3 du Code civil français, par réciprocité, la jurisprudence déduit que «toute personne étrangère, quel que soit son lieu de résidence est soumise pour son statut personnel à la loi du pays dont elle possède la nationalité ». Toutefois, si l'étranger estautorisé à demander le bénéfice de sa loi personnelle, cette loi étrangère considérée non conforme aux exigences de l'ordre public international peut être évincée partiellement ou totalement.

\footnotetext{
8 - Loi n 70-03 Bulletin Officiel n5358 du 2 ramadan 1426 (6 octobre 2005).

9 - Guide pratique du Code de la famille marocaine. Ministère de la justice. Royaume du Maroc. Imprimerie de Fédala, $1^{\text {ère }}$ édition février 2005, p.38.

10 - Le Coran est bien clair que s'il y a risque d'injustice du mari à l'égard de ces épouses, la polygamie est rejetée. Le verset 3 dans Sourate les femmes précise «... il est permis d'épouser deux, trois ou quatre, parmi les femmes qui vous plaisent, mais si vous craignez de n'être juste pas avec celles-ci, alors une seule».

يقول الله تعالى : " و ان خفتم ألا تقسطو افي اليتامى فانكحو ا ما طاب لكم من النساء مثنى و ثلاث و رباع فإن خفتم ألا تعدلو ا فو احدة أو ما ملكت أيمانكم

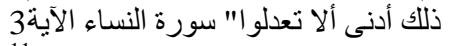

${ }^{11}$ - Rajaâ NAJI El MEKKAOUI. ; «La Moudawana, le référentiel et le conventionnel en harmonie- De la réforme de la Moudawana à la concrétisation de son âme».Tome 3. Edition et impression Bouregreg, Rabat, révisée2009, p.63.

12. Article 433-20 du Code pénal français, modifié par Ordonnance n²000-916 du 19 septembre 2000 - Art. 3 (V) JORF 22 septembre 2000, en vigueur le $1^{\text {er }}$ janvier 2002.

13 -M.LAMARCHE. ; «Ne confondons pas polygamie et bigamie! Distinguons union légale et union de fait », Dr. Fam. 2010, alerte n47, p.3.
} 
Ainsi, les solutions juridiques posées par le droit international privé permettent justement de ne retenir que les dispositions compatibles avec le respect des droits fondamentaux de l'individu, sans toutefois renier son appartenance à une civilisation autre qu'occidentale.

\section{II-La Polygamie Marocaine Face Au Droit International Français A-Convention franco-marocaine du 10 août $1981^{14}$}

La France et le Maroc ont signé une Convention en date du 10 août $1981^{\mathbf{1 5}}$ dite "loi Pasqua" relative au statut familial et à la coopération judiciaire au vu des relations entre les ressortissants des deux États et de la nécessité de conserver aux personnes les principes fondamentaux de leur identité nationale.

Convention qui a non seulement pour but de protéger et de renforcer des liens existants ou susceptibles de se créer entre les ressortissants des deux États mais aussi d'établir des règles communes de gestion de conflit des lois et de juridictions relatives au statut familial.

Gestion de deux types de statuts personnels complétement antagonistes : polygamie, répudiation,empêchement au mariage en raison del'allaitement sont tous prévus dans le droit marocain, alors qu'en droit français, ils sont inconnus et ne peuvent être admis dans l'ordre juridique français pour leurs contrariétés à l'ordre public international français. Parallèlement, des institutions comme l'adoption, la reconnaissance d'enfant naturel, la légitimation, sont réglementées par le droit français mais ne peuvent être admises par les tribunaux marocains pour leur contrariété également à l'ordre public marocain.

En effet, la ratification de cette Convention est considérée comme une solution permettant une meilleure coordination entre les deux systèmes.Mais, pour atteindre l'harmonisation des solutions, il faut d'abord que l'ordre juridique d'accueil fasse preuve, afin de se montrer respectueux de ladite Convention, d'un traitement tolérant dans la réception de la loi étrangère.Or, ceci n'est vraiment pas une simple tache où l'institution d'accueil n'accepte aucune indulgence transgressant ces valeurs fondamentales.

Il est vrai que chaque État est souverain de fixer les limites à la réception des droits étrangers lorsqu'ils risquent de heurter les fondements et les principes de la société d'accueil, cependant, il ne s'agit pas d'être rigide, mais il s'avère importantde trouver une solution souple et médiane qui réponde à ce rôle d'harmonisation et de coordination qu'est censée apporter la Convention du 10 août 1981 dans laquelle la France qu'est la société d'accueil doit gérer les unions polygamiques marocaines résidentes sur son territoire.

Par ailleurs, Il y a lieu de signaler que le juge français s'oppose à toute règle instaurant des empêchements au mariage de nature religieuse, ce qui montre clairement la non efficacité de la Convention franco-marocaine.

L'empêchement religieux exigé par le droit marocain interdisant tout mariage entre une ressortissante marocaine musulmane et un non musulman est incontestablement contraire aux valeurs fondamentales françaises touchant la liberté matrimoniale. ${ }^{\mathbf{1 6}}$

La Cour d'appel de Paris dans une décision rendue le 9 juin $1995^{17}$ à l'occasion d'une affaire où le mari français prétendait que son mariage avec une Marocaine avait été contracté dans le seul but de permettre à cette dernière d'acquérir la nationalité française. Il a voulu prouver la nullité de son mariage en s'appuyant sur le droit marocain qui prohibe le mariage entre une Marocaine musulmane et un non-musulman.

${ }^{14}$ - Bulletin Officiel no 3910 du mercredi 7 octobre 1987. Dahir n 1-83-197 du 11 rebia I 1407 (14 novembre 1986) portant publication de la Convention entre le Royaume du Maroc et la République française relative au statut des personnes et de la famille et à la coopération judiciaire. Rabat le 10 août 1981.

${ }^{15}$-Voir : F.MONEGER., « La Convention franco-marocaine du 10 août 1981 relative aux statuts des personnes et de la famille et à la coopération judiciaire ».Rev. crit. DIP.1984, p. 29 et s ; P. DECROUX. ; «La Convention franco-marocaine du 10 août 1981 relative au statut des personnes et de la famille et à la coopération judiciaire», J.D.I, 1985, p. 66.

16 - D. HOLLEAUX et J. FOYER, G. GEOUFFRE de LA PARADELLE., «Droit international privé ». Masson, 1987, $\mathrm{n}^{\circ}$ 1141; Y. LOUSSOUARN, P. BOUREL., «Précis de droit international privé ». Dalloz, 2004, $8^{\text {ème }}$ édition.

17 - C. A. Paris, 9 juin 1995, D. 1996. Somm. comm., p. 171, observation B. AUDIT. 
À la lecture de cette décision, on décèle que les réponses apportées par la Convention franco-marocaine du 10 août 1981 demeurent insuffisantes et inefficaces quant à l'harmonisation des solutions.

Faut-il comprendre que les juridictions marocaines déclarent la loi française incompatible avec l'ordre public national marocain et vice versa?

Pour ce faire, les juridictions marocaines peuvent se fonder sur l'article 4 de ladite Convention qui précise que « $l \boldsymbol{a}$ loi de l'un des deux États désignés par la présente Convention ne peut être écartée par les juridictions de l'autre État que si elle est manifestement incompatible avec l'ordre public ».

Ainsi, la Convention franco-marocaine du 10 août 1981 a montré parfaitement un exemple de défaillance voire d'échecdans son rôle d'harmonisation et de coordination.

\section{B-Le droit applicable à la polygamie en France}

1-La célébration de la polygamie en dehors du territoire d'accueil

Il se peut que le mari marocain, même domicilié en France, se déplace au Maroc et y contracte une nouvelle union conformément à sa loi nationale. Cette seconde union serait-elle valable en France étant donné qu'elle a été contractée dans le pays d'origine et en conformité avec le statut personnel de l'époux ?

Du point de vue pénal, l'épouse ne peut pasobtenirgain de cause car la polygamie n'a pas été contractée sur le territoire français. Cependant elle peut, sur le plan civil, obtenir le divorce dans la mesure où ce second mariage rend intolérable le maintien du lien conjugal.

\section{2-Interdiction de contracter un mariage polygamique en France}

Il est formellement interdit de célébrer un mariage polygamique en France, même si les deux parties sont de statut personnel polygamique et cela concerne également les ressortissants marocains qui sont régit par le Code de la famille marocaine de 2004, une institution inconnue du droit français.

La jurisprudence a abordé le problème que soulève la reconnaissance de ce type de mariage particulier «avec un effort de compréhension et sans trop de préjugés ${ }^{18}$, elle a même pu le cerner. La doctrine de son côté a soulevé que "l'union des sexes est un problème commun à toute l'humanité, les solutions qu'il reçoit peuvent être classées dans la catégorie "mariage" malgré la différence notable qui les sépare de notre conception, et sans que nous les regardions comme équivalentes moralement $\gg .{ }^{19}$

Toutefois, Il demeure quel'ordre public français est d'une telle ténacité, qu'il ne permet pas la reconnaissance du statut personnel des ressortissants des pays étrangers, par contre il accepte uniquement quelqueseffetsproduites parles mariages polygamiques.

En somme, la célébration en France du mariage polygame ne peut donc en aucun cas avoir lieu, même si les parties au contrat manifestent clairement leurs consentements (époux et les deux épouses). Une protection de la première épouse contre l'introduction dans sa vie conjugale d'une coépouse justifié par la nécessité de préserver de l'ordre public. $^{20}$

3-La nullité du mariage contracté par une Française et un étranger polygame

Un arrêt de la Cour de Cassation a été rendu le 24 septembre 2002 prononçant la nullité du mariage entre une Française célibataire et un musulman polygame à l'étranger. ${ }^{21}$

18 - P.MAYER. ; «Conflit de civilisation et droit international privé, Polygamie et répudiation ». Librairie Droz, Genève, 1972, p.32.

19 - H. BATIFFOL. ;《Traité élémentaire de droit international privé ». Paris, LGDJ, 1955, $2^{\mathrm{ème}}$ édition, $\mathrm{n}{ }^{\circ} 434$.

20 - T.G.I Paris le 22 janvier 1968, Mpouna c. dame Mpouna, J.C.P. 1968.IV.116, R.1969, Somm. 775,Clunet, 1969, 406, note Khan.

21 - C. Cass 1ère Ch. Civ., 24 septembre 2002, pourvoi no 00-15.789, Bull. civ. I, no 214; R., p. 339; D. 2003. Somm. 1935, obs. J.-J. Lemouland ; JCP 2003. II. 10007, conclusion. Sainte-Rose, note Devers; Defrénois 2002.1467, obs. J. Massip ; AJ Fam. 2002. 384, obs. S. D.-B ; RJPF 2003-1/18, note M. Oudin ; Dr. et patr., janv. 2003, p. 120, obs. 
Était en cause une demande en nullité d'un mariage entre un Libanais et une Française, célébré au Caire en 1994, alors que l'époux était encore lié par un mariage avec une première épouse de nationalité libanaise datant de 1985. Saisis par le procureur de la République près du Tribunal de grande instance de Nanterre, les juges du fond ont considéré que l'union célébrée en Égypte était dépourvue d'effet en France. Rejetant le pourvoi formé par le mari et son épouse française, la Cour de cassation a approuvé la qualification retenue par la Cour d'appel de Versailles de l'empêchement au mariage édicté par l'article 147 du Code civil. Cette disposition qui énonce "qu'on ne peut contracter un second mariage avant la dissolution du premier mariage », contient un empêchement qualifié de bilatéral et absolu.

Par conséquent, l'article 147 du Code civil interdit non seulement à un conjoint français de conclure un second mariage avant la dissolution du premier mais également à une Française célibataire d'épouser un homme déjà marié. La sanction du non-respect de l'empêchement à mariage est la nullité absolue pour bigamie qui entraîne l'annulation de la seconde union dès son origine, sans possibilité de régularisation a posteriori comme l'indique une décision de la Cour d'appel de Grenoble du 23 janvier 2001. ${ }^{22}$

\section{III- La Limitation Des Effets De La Polygamie Par Le Droit Des Etrangers Et Ces Consequences A- La limitation des effets de la polygamie}

Malgré que la polygamie ne soit pas « légalisée » en France, les règles du droit international privéont uniquement admis les effets découlant de la polygamiesur le territoire nationalpour la liquidation du régime matrimonial,pourpermettre à une seconde épouse d'obtenir une pension alimentaire, pourbénéficier d'une indemnisation ou encore pourprétendre à des droits successoraux. ${ }^{23}$ En effet, La Cour de cassation a rappelé à maintes reprises que deux femmes et leurs enfants respectifs pouvaient venir concurremment à la succession du mari polygame sans que l'ordre public s'en trouve heurté. Cette position a été adoptée dès 1979 dans l'affaire Bendeddouche. $^{24}$

La loi n 93-1027 du 24 août 1993(dite loi Pasqua)relative à la maîtrise de l'immigration et aux conditions d'entrée, d'accueil et de séjour des étrangers en France a considérablement réduit le nombre de problèmes relatifs aux effets des unions polygamiques susceptibles de se présenter devant le juge français ${ }^{25}$ en prévoyant des mesures de restriction pour le regroupement familial et une réglementation pour l'entrée et le séjourqui a fait de la polygamie un motif de retrait ou de non renouvellement du titre de séjour.

\section{1-Restriction du regroupement familial}

Le regroupement familial a d'abord été réglementé par des accords bilatéraux, tel que l'accord franco-algérien de $1968{ }^{26}$ le décret 76/383 du 29 avril 1976 dans lequel le gouvernement a instauré un véritable droit au séjour au bénéfice de la famille «immédiate » du travailleur étranger.

L'installation de familles polygames en France a d'abord été admise par un le fameux arrêtMontcho ${ }^{27}$ du 11 juillet $1980^{28}$ dans lequel le Conseil d'État a autorisé le regroupement familial au profit d'une seconde épouse et que le fait de faire venir une seconde épouse ne heurtait pas l'ordre public français.

F. Monéger; LPA 15 oct. 2003, note C. Brière; RTD civ. 2003. 62, obs. J. Hauser; Rev. crit. DIP 2003. 271, note B. Bourdelois.

${ }_{22}$ - Grenoble, 23 janvier 2001, Juris-data, n 2001-150549; Dr. Fam. 2002, comm. no 54, obs. H. Lécuyer.

23 - Cours d'appel de Paris du 8 Novembre 1983, affaire Kaci et C. MAHMOUDI.

24 - Cass.Civ. 3 janvier 1980 (et 18 décembre 1979), D 1980, p 549 note POISSON-DROCOURT, GAJDIP ANCEL et LEQUETTE, ${ }^{\circ} 61$ p.573 ; JDI 1980, p 337, note SIMON-DEPITRE ; décision de la CA de Paris pour l'affaire Bendeddouche, RCDIP 1978, p.507, note BATIFFOL ; dans le même sens : Paris 8 novembre 1983, RCDIP 1984, p. 18, note LEQUETTE, Rép. Not. 1984, art 33284, note REVILLARD, JDI 1984, p 881, note SIMON-DEPITRE.

25 - P. LAGARDE «La théorie de l'ordre public international face à la polygamie et à la répudiation. L'expérience française » in Nouveaux itinéraires en droit Hommage à F. RIGEAUX, éditionBruylant, Bruxelles 1993, p.208.

${ }^{26}$ - Voir Le regroupement familial en droit comparé. Toute une série d'accords bilatéraux vont ainsi être conclus à cette époque, des pays du Maghreb à l'Afrique noire, mais aussi l'Espagne et le Portugal, Voir LABAYLE.H, «Le droit de l'étranger au respect de sa vie familiale », RFDA 1993, p.514.

27 - CE 11 juillet 1980, nº 16596, arrêt Montcho, publié au recueil Lebon.

28 - Rev. crit. DIP 1981 p 658, note BISCHOFF, JCP éd. G. 1981, II, p 19629, AJDA 1980 conclusion ROUGEVINBAVILLE, $\mathrm{p} 548$, note FEVER PINAULT. 
Le juge administratif s'est placé non du point de vue de l'ordre public français mais de celui de la loi personnelle des intéressés et préserver l'unité de la cellule familiale et la vie familiale effective qui en découlait.

Il ne faut pas oublier que le Conseil d'État deux ans avant de prononcerl'arrêt Montcho, avait reconnu dans l'arrêt GISTI du 8 décembre 1978 que le droit à une vie familiale normale était un principe général du droit découlant du Préambule de la Constitution de 1946 selon lequel «la nation assure à la famille les conditions nécessaires à son développement et garantit, notamment à l'enfant et à la mère, la sécurité matérielle » et impliquait pour les étrangers le droit d'être rejoint par leur famille.

Cette décision était donc incontestable car elle permettait la protection d'un droit fondamental, mais aussi parce qu'elle respectait la démarche classique en droit international privé de la qualification en sous-ordre. L'arrêt Montcho fut très contesté et donna lieu à un véritable débat politico-juridique.

Quelques années plus tard, la loi n 93-1027 du 24 août 1993 relative à la maitrise del'immigration et aux conditions d'entrée, d'accueil et de séjour des étrangers enFrance ainsi que la directive européenne du 22 septembre 2003 sur le regroupement familial ont intervenu pour limiter au maximum voire mêmemettre fin àune polygamie existante surle territoire français.

En effet, le Marocain polygame ne pouvait plus ramener la seconde épouse sur le territoire français c'est-à-dire bénéficier du regroupement familial mais il pouvait imposer à la première épouse son deuxième mariage célébré au Maroc ainsi que ses effets en France.

Ainsi, la loi du 24 août 1993 a formulé l'interdiction du regroupement familial pour une seconde épouse et les enfants nés de celle-ci. L'époux qui faisait venir une seconde conjointe avec ses enfants pouvait être sanctionnée par le retrait de son titre de séjour. D'ailleurs, l'attitude prise par la loi du 24 août 1993 a toujours été la position de l'ordonnance du 2 novembre 1945 qui prévoit dans son article 30 que: «lorsqu'un étranger polygame réside sur le territoire français avec un premier conjoint, le bénéfice du regroupement familial ne peut être accordé à un autre conjoint. Sauf si cet autre conjoint est décédé ou déchu de ses droits parentaux, ses enfants ne bénéficient pas non plus du regroupement familial».

Le Conseil constitutionnelen France, dans sa décision du 13 août $1993^{29}$ adopte une position similaire à celle de la loi Pasqua dans la mesure où il considéréque «les conditions d'une vie familiale normale sont celles qui prévalent en France, pays d'accueil, lesquelles excluent la polygamie ; que dès lors les restrictions apportées par la loi au regroupement familial des polygames et les sanctions dont celles-ci sont assorties ne sont pas contraire à la Constitution $»{ }^{30}$

Il y a lieu de souligner que toutes ces positions prises par la loi Pasqua, la directive européenne de 2003 ainsi que le Conseil constitutionnel répondent favorablement aux politiques d'immigration qui depuis plusieurs années œuvraient à limiter la venue des étrangers en France. Le regroupement familial, expression du droit fondamental de mener une vie familiale normale comme il a été avancé par l'arrêt Montcho devint donc un droit conditionné. L'objectif d'intégration de la politique d'immigration justifia notamment l'exigence de la condition de monogamie.

\section{2-Retrait de la carte de séjour}

L'article 8 de la loi Pasqua du 24 août 1993 prévoit l'impossibilité de délivrer une carte de résident ou, le cas échéant, le devoir de la retirer aux étrangers vivant en état de polygamie. Cette loi a fait entrer, pour la première fois, dans l'Ordonnance du 2 novembre $1945^{31}$, des dispositions relatives à la polygamie.

C'est ainsique l'article 15 bis de l'Ordonnanceinterdit la délivrance d'une carte de résident à un étranger vivant en état de polygamie ou à son conjoint en prévoyant clairement que « ... la carte de résident ne peut être délivrée à un

${ }^{29}$ - Cons.const. 13 août 1993, RCDIP, 1993, p. 597. Sur le problème de l'atteinte au caractère fondamental de ce droit : VANDENDRISSCHE.X. ; « Le droit à la vie familiale est-il un droit fondamental ? », in Le droit de la famille à l'épreuve des migrations transnationales, LGDJ, 1993, p. 54.

30 - Constitution 1977.

31 - Ordonnance $\mathrm{n}^{\circ}$ 45-2658 du 2 novembre 1945 relative aux conditions d'entrée et de séjour des étrangers en France. 
ressortissant étranger qui vit en état de polygamie ni aux conjoints d'un tel ressortissant. Une carte de résident délivrée en méconnaissance de ces dispositions doit être retirée ».La situation de polygamie effective fait donc obstacle à la délivrance de la carte de résident quel que soit le titre dont peut se prévaloir l'étranger, même s'il est parent d'un enfant français.

De son côté, l'article 30 de l'Ordonnance interdit le regroupement familial polygamique en France et prévoit même le retrait du titre de séjour s'il est « sollicité ou obtenu par un autre conjoint est, selon le cas, refusé ou retiré. Le titre de séjour du ressortissant étranger polygame quia fait venir auprès de lui plus d'un conjoint, ou des enfants autres que ceux du premier conjoint ou d'un autre conjoint décédé ou déchu des droits parentaux ${ }^{32}$ se verra le titre de séjour retiré.

De surcroît, la loi n 93-1027 du 24 août 1993 énonce que la deuxième épouse qui était déjà sur le territoire français nepouvait plus bénéficier du renouvellement de son titre de séjour. Beaucoup desecondes épouses se sont ainsi retrouvées en situation irrégulière, exception faite àl'époque pour les épouses ayant des enfants français, ou résidant en France depuisplus de 15 ans, qui ont pu bénéficier d'un titre de séjour selon les dispositions dela circulaire du 8 février $1994 .^{33}$

Il a en effet été jugépar le juge administratif dans l'arrêt G.I.S.T.I que « la protection des droits acquis par la délivrance de la carte de résident obtenue antérieurement à 1993 ne joue plus quand il s'agit de demander le renouvellement de la carte [...], l'administration pouvant légalement refuser le renouvellement de la carte de résident d'un ressortissant vivant en état de polygamie ou de ses conjoints quelle que soit la date de délivrance de ce titre de séjour $\gg{ }^{34}$

Les juges dans cet arrêtne s'appuient pas sur l'article 37 de l'Ordonnance qui prévoit que le retrait ne s'applique « qu'à des étrangers ayant reçu un titre de séjour après l'entrée en vigueur de la loi du 24 août 1993 », mais ils adoptent une attitude rigide qui répond parfaitement à l'esprit de la loi 1993.

Il y a lieu de souligner que la loi de 1993 est sévère, radicale dans ces propos.La carte de résident ne peut être délivrée à un étranger vivant en état de polygamie. La formulation est stricte et conçue de telle manière que les autorités administratives ne disposent d'aucun pouvoir d'appréciation.

La loi du 24 août 1993 a marqué une volonté ferme de mettre fin au droit du regroupement familial aux marocains polygames, mais également a montréune volonté de s'abstenir de leur octroyer ou de leur renouveler le titre de séjour.

\section{B-LesConsequences Des Effets De LaDe La Polygamie}

1- Suppression d'un des effets principal de l'union polygamique

De ce qui précède, il a été constaté que la règle de conflit donne droit aux unions polygamiques valablement contractées à l'étranger de produire leurs effets en France. Certes, en empêchant les couples polygames de vivre ensemble, l'effet principal de cette union est bafoué.« La condition première et fondamentale de la reconnaissance en France des mariages polygamiques valablement célébrés à l'étranger » a disparu est ce depuis 1993 avec l'arrêt Montcho.

\section{2-Suppression d'autres effets de la polygamie}

Théoriquement et grâce au droit international privé, la seconde épouse, retournée ou restée dans son pays d'origine, bénéficie toujours d'un certain nombre de droits qui lui ont été reconnus, tels que le droit de succession, le droit d'indemnisation ou le droit à une pension alimentaire. Mais sur le plan pratique lui est-il possible de faire valoir ces droits?

\footnotetext{
32 -Article 30 del'Ordonnance du 2 novembre 1945.

33 - Extrait de la circulaire du 8 février 1994 d'application de la loi du 24 août 1993 : « les dispositions des articles 15 bis et 16 de l'Ordonnance ne s'opposent pas à la délivrance d'une carte de séjour temporaire aux étrangers vivant en France en état de polygamie ou à leurs conjoints. Il convient pour ce faire de vérifier que les personnes en cause satisferont aux conditions d'une admission au séjour en qualité de résident temporaire.

34 - Arrêt GISTI, 18 juin 1997, req. N 162517, classé en B ; C.A.A Douai 12 novembre 2002, AJDA, 28 avril 2003, p.851.
} 
Logiquement, il lui est impossible de revendiquer ces droits si elle ne dispose pas de carte de séjour ou qu'elle lui a été retirée et qu'elle ait été reconduite à son pays d'origine !!!

Autre problème qui empêche que les effets de cette union se produisent est certainement l'absence d'information. En effet, Dans son rapport ${ }^{35}$, le Haut conseil à l'intégrationsignale que "cette matière reste trop peu connue des femmes concernées et peut-être aussi des intervenants sociaux qu'elles rencontrent ou côtoient».

En l'absence d'information, la seconde épouse ne pourra pas bénéficier des effets de son union conjugale et verra forcément ces droits éclaboussés d'où une non protection de ces familles polygames.

\section{Conclusion:-}

Au terme de cette réflexion, nous pouvons conclure que la réception de la polygamie par l'ordre juridique français n'est pas évidente. Un ordre juridique qui reste attaché aux principes fondamentaux de la loi nationale, un ordre juridique qui n'a pas respecté la culture et l'identité des marocains résidents enFrance enlimitant la polygamie par la restriction du regroupement familial et le retrait ou le non renouvellement de la carte de séjour.

Même la Convention franco-marocaine qui est censée apporter des solutions,assurer une harmonisation et un rapprochement entre deux systèmes antagonistes a perdu de plus en plus son sens dans la mesure oùl'harmonie du statut personnel des étrangers polygames en reste néanmoins ébranlée, c'est ce qui nous pousse réellement à nousinterroger sur l'utilité réelle de cette Convention et de nous demander si cette harmonisation est possible tant que chaque partie insiste sur ses positions.Peut-être la solution serait-elle la nécessité de créer un nouvel outil plus efficace pour harmoniser les deux systèmes !!

Un problème qui se pose également pour les effets du mariage marocain qui ont que peu de place sur le sol français. On assistera donc rapidement au déclin des effets des unions polygamiques en France mais aucune réforme n'a été entreprise pour adapter le droit international privé à cette réalité d'où la nécessité d'une réforme garantissant une protection pour la famille polygamique en l'occurrence les femmes et les enfants.

Pour terminer, je dirai que les Marocains résidents à l'étranger se trouvent face à un double défi avant de penser à fonder un ménage polygamique : l'autorisation du juge marocain et le rejet de l'union sur le territoire français. Ce double obstacle servira certainement à la fin des unions polygamiques, au moins pour les Marocains se frottant directement avec la France.

\section{Bibliographie:- \\ Ouvrages}

1. BATIFFOL.H.; «Traité élémentaire de droit international privé ».Paris, LGDJ, 1955, $2^{\mathrm{ème}}$ édition, ${ }^{\circ} 434$.

2. CARBONNIER. J.; "Terre et ciel dans le droit français du mariage », in mélanges Ripert, Dalloz, 1950.

3. DECROUX.P. ; "La Convention franco-marocaine du 10 août 1981 relative au statut des personnes et de la famille et à la coopération judiciaire », J.D.I, 1985.

4. HOLLEAUX. D et J. FOYER, G. GEOUFFRE de LA PARADELLE. ; «Droit international privé ». Masson, $1987, \mathrm{n}^{\circ} 1141$.

5. KISAAKYE. ESTHER M.; «Women, Culture and HumanRights: FemaleGenital Mutilation, Polygamy and Bride Price » dans Wolfgang Benedek, Londres, World University Service, 2002.

6. KISAAKYE. ESTHER M. et GERDOberleitner, dir.; «The HumanRights of Women: International Instruments and AfricanExperiences », Londres, World University Service, 2002.

7. LABAYLE.H ; «Le droit de l'étranger au respect de sa vie familiale ». RFDA, 1993.

8. LAGARDE.P.; "La théorie de l'ordre public international face à la polygamie et à la répudiation. L'expérience française » in Nouveaux itinéraires en droit Hommage à F. RIGEAUX, Ed. Bruylant, Bruxelles 1993.

9. -LAMARCHE.M.; «Ne confondons pas polygamie et bigamie! Distinguons union légale et union de fait ». Dr. Fam. 2010, alerte ${ }^{\circ} 47$.

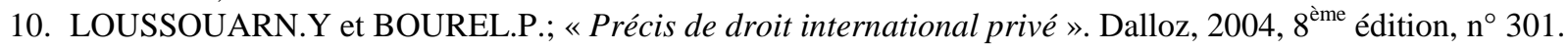

\footnotetext{
35 -Voir son récent rapport intitulé : «Les droits des femmes issues de l'immigration ». Avis au Premier Ministre du
} 2 juillet 2002, p. 8 ; http://www.cfdt.fr/actu/societe/societe_081.htm. 
11. MAYER.P.; «Conflit de civilisation et droit international privé, Polygamie et répudiation ». Librairie Droz, Genève, 1972.

12. MONEGER.F.; «La Convention franco-marocaine du 10 août 1981 relative aux statuts des personnes et de la famille et à la coopération judiciaire ». Rev. crit. DIP.1984.

13. NAJI El MEKKAOUI.Rajaâ. ; «La Moudawana, le référentiel et le conventionnel en harmonie - De la réforme de la Moudawana à la concrétisation de son âme ». Tome 3. Edition et impression Bouregreg, Rabat, révisée 2009.

14. STRASSBERG Maura I.; «The Challenge of Post-ModernPolygamy: ConsideringPolyamory » (2003) 31 Capital U.L. Rev. 439.

15. VANDENDRISSCHE.X. ; «Le droit à la vie familiale est-il un droit fondamental ? », in Le droit de la famille à l'épreuve des migrations transnationales, LGDJ, 1993.

\section{Textes Juridiques:-}

1. Bulletin Officiel $n^{\circ} 3910$ du mercredi 7 octobre 1987. Dahir $n^{\circ}$ 1-83-197 du 11 rebia I 1407 (14 novembre 1986) portant publication de la Convention entre le Royaume du Maroc et la République française relative au statut des personnes et de la famille et à la coopération judiciaire, Rabat le 10 août 1981.

2. Code civil français - Livre $1^{\mathrm{er}}$ des personnes, titre $\mathrm{V}$ du mariage. Créé par Loi n ${ }^{\circ} 1803-03-17$ promulguée le 27 mars 1803.

3. Code pénal- nouveau Code pénal. Dalloz, 1992-1993.

4. Code pénal français, modifié par Ordonnance n²000-916 du 19 septembre 2000.

5. Dahir $\mathrm{n}^{\circ} 1-04-22$ Du 12 hija 1424 (3 Février 2004) portant promulgation de la loi $\mathrm{n}^{\circ} 70-03$ portant Code de la famille.

6. Directive européenne du 22 septembre 2003 sur le regroupement familial.

7. Guide pratique du Code de la famille marocaine. Ministère de la justice. Royaume du Maroc. Imprimerie de Fédala, 1 ère édition février 2005.

8. Loi $\mathrm{n}^{\circ} 70-03$ - Bulletin Officiel $\mathrm{n}^{\circ} 5358 \mathrm{du} 2$ ramadan 1426 (6 octobre 2005).

9. Loi n ${ }^{\circ}$ 93-1027 du 24 août 1993 (dite loi Pasqua) relative à la maîtrise de l'immigration et aux conditions d'entrée, d'accueil et de séjour des étrangers en France.

10. Ordonnance $\mathrm{n}^{\circ} 45-2658$ du 2 novembre 1945 relative aux conditions d'entrée et de séjour des étrangers en France. 